\title{
INHIBITION OF BOTH PHYSIOLOGICAL AND ABERRANT WNT/B- CATENIN SIGNALING ACTIVITIES ASSOCIATED WITH STEMNESS IN NASOPHARYNGEAL CARCINOMA
}

Yue Cheng, Yee Peng Phoon, and Maria L. Lung

University of Hong Kong, Pokfulam, Hong Kong.

We previously demonstrated that basic or physiological levels of $\beta$-catenin signaling and tissue context play decisional roles in the regulation of self-renewal networks in nasopharyngeal carcinoma (NPC) HONE1 cells. Introduction of physiological levels of $\beta$-catenin signaling in HONE1 hybrid cells plays a central role in the control of other pathway activities, such as TGF- $\beta$, Activin, and pluripotency maintenance (LIFR and IL6ST), during the stemness transition process. These results revealed novel regulatory relationships among Wnt signaling, stemness, epithelial-mesenchymal transition (EMT), tumor suppressive pathways and expression of common cancer stem cell surface markers, such as CD44 and CD133, in human tumor cells. In current studies, we found that several NPC cell lines, including CNE1, HK1, HNE1, SUNE1, and C666-1, have aberrant and significant up-regulation of $\beta$-catenin expression, when compared to HONE1 cells that have low levels endogenous $\beta$-catenin expression. In these NPC cells with aberrant $\beta$ catenin signaling activities, the Wnt pathway (Axin2, APC, TCF1 and TCF3), stemness factors (Nanog, and Oct4) and pluripotency markers (IFITM1 and GRB7) are strongly overexpressed, compared to HONE1 cells. Our findings suggest that aberrant Wnt/Bcatenin signaling activities may play a prominent role in promoting tumor progression in NPC cells. To further verify the role of aberrant $\beta$-catenin signaling in NPC, we utilize shRNA to knock down $\beta$-catenin expression in these cells, including CNE1 and HK1. As found in HONE1 hybrids, the diminished expression of core stem cell factors (Nanog, Oct4, Klf4, and Sox2) is also detected in both CNE1 and HK1 cell lines. Concomitantly, the gene expression of EMT markers (E-cadherin and Zeb1) is also inhibited when $\beta$ catenin is knocked down by shRNA $\beta$-catenin infection. These preliminary results suggest that $\beta$-catenin signaling plays a dominant role in regulating both physiological and aberrant Wnt/ $\beta$-catenin signaling activities as well as EMT events in NPC development. 